\title{
ANALYSIS OF RADOME COVERED CIRCULAR REFLECTORS BY COMPLEX SOURCE-DUAL SERIES APPROACH
}

\section{Taner Oğuzer*, Ayhan Altıntas}

Department of Electrical and Electronics Eng., Bilkent University 06533 Bilkent, ANKARA, TURKEY

Alexander.I.Nosich

Currently Visiting Prof. at Department of EE-CS, Kumamoto University Kumamoto 860, JAPAN

\section{Introduction}

Radiation from a two dimensional reflector antemna covered by a cylindrical ralome is analyzed by complex source-dual series approach. It is only performed for the electrically polarized incident field. The approach is not based on the moment method but on the analytical-numerical type regularization technique. The method gives the exact solution with any desired accuracy and the directivity of the feed antenna can be modelled by using the complex source method [1]. In numerical results, the far field radiation patterns are obtained and the effect of the radome is verified.

\section{Formulation}

The circular reflector and the radome geometry is shown in Figure 1. The reflector is modelled by a part of a circular, zero-thickness, perfectly conducting material of the radius a and angular wilth $2 \theta_{a p}$. A complex line source is located at the focal point $\left(r_{o}=a / 2, \phi_{o}=0\right)$. It simulates the physical directivity of a feed antenna by the directivity factor $k b$ ( $k$ is wavenumber). The problem is two dimensional in geometry and the radome is a co-axial dielectric cylinder of inner radius $c$, onter radius $h$, thickness $d=h-c$ and the relative permitivity is $t_{r}$.

The requirements for the rigorons solution of the present boundary value problem can be stated as the satisfaction of the Helmhlotz wave equation, Sommerfeld radiation condition, edge conditions and the boundary conditions for the dielectric and conducting boundaries.

The total electric field expansions in the four regions can be written as

$$
E_{z}^{T}=\sum_{n=-\infty}^{\infty}\left\{\begin{array}{rl}
A_{n} J_{n}\left(k_{o} r\right)+b n^{2} & r<a \\
B_{n} J_{n}\left(k_{o} r\right)+C_{n} H_{n}^{(1)}\left(k_{o} r\right) & a<r<c \\
D_{n} J_{n}(k \cdot r)+F_{n} H_{n}^{(1)}(k r) & c<r<c+d \\
G_{n} H_{n}^{(1)}\left(k_{o} r\right) & c+d<r
\end{array}\right\} e^{i n \phi}
$$


where $k=k_{o} \sqrt{\mu_{o} \epsilon_{\mathrm{r}}}, k_{o}$ is free space wavenumber, $\phi=\pi-\varphi$ and the incident field expansion coefficient is given as $b_{\mathfrak{n}}^{E}=J_{n}\left(k_{o} r_{s}\right) H_{n}^{(1)}\left(k_{o} a\right) e^{-i n \theta_{s}}$ where $\theta_{s}$ and $r_{s}$ is defined in [3].

Bunndary conditions are imposed at the dielectric boundaries at $c$ and $c+d$, and further at the metal and slot part of the circular interface $r=a$. Then, the following dual series equations are obtained in terms of the inner region expansion coefficient.

$$
\begin{gathered}
\sum_{n=-\infty}^{\infty} x_{n}\left\{\frac{1}{J_{n}\left(k_{o} a\right) H_{n}^{(1)}\left(k_{o} a\right)}-\frac{\gamma_{n}}{J_{n}^{(1)}\left(k_{o} a\right)}\right\} e^{i n \phi}=\sum_{n=-\infty}^{\infty} \frac{J_{n}\left(k r_{s}\right)}{J_{n}\left(k_{o} a\right)} e^{i n\left(\phi-\theta_{s}\right)} ; \phi \in S \\
\sum_{n=-\infty}^{\infty} x_{n} e^{i n \phi}=0 ; \quad \phi \in M \\
\gamma_{n}=\frac{h_{n}}{J_{n}\left(k_{o} a\right) h_{n}+g_{n} H_{n}^{(1)}\left(k_{o} a\right)}
\end{gathered}
$$

where $x_{n}=A_{n} J_{n}\left(k_{o} a\right)+b_{n}^{E}, g_{n}=\beta_{n} a_{n}-\alpha_{n} b_{n 2}$ and $h_{n}=-\beta_{n} c_{n}+\alpha_{n} d_{n}$. Further, $\alpha_{n}, \beta_{n}, a_{n}, b_{n}, c_{n}$ and $d_{n}$ are defined as follow.

$$
\begin{gathered}
\alpha_{n}=H_{n}^{(1)^{\prime}}\left(k_{o} h\right) J_{n}(k h)-\sqrt{\frac{\epsilon_{r}}{\mu_{r}}} J_{n}^{\prime}(k h) H_{n}^{(1)}\left(k_{o} h\right) \\
\beta_{n}=H_{n}^{(1)}(k \cdot h) H_{n}^{(1)^{\prime}}\left(k_{o} h\right)-\sqrt{\frac{\epsilon_{r}}{\mu_{r}}} H_{n}^{(1)^{\prime}}(k \cdot h) H_{n}^{(1)}\left(k_{o} h\right) \\
a_{n}=J_{n}(k \cdot c) \cdot J_{n}^{\prime}\left(k_{o} c\right)-\sqrt{\frac{\epsilon_{r}}{\mu_{r}}} J_{n}\left(k_{o} c\right) \cdot J_{n}^{\prime}(k c) \\
b_{n}=J_{n}^{\prime}\left(k_{o} c\right) H_{n}^{(1)}(k \cdot c)-\sqrt{\frac{\epsilon_{r}}{\mu_{r}}} J_{n}\left(k_{o} c\right) H_{n}^{(1)^{\prime}}(k c) \\
c_{n}=J_{n}(k c) H_{n}^{(1)^{\prime}}\left(k_{o} c\right)-\sqrt{\frac{\epsilon_{r}}{\mu_{r}}} H_{n}^{(1)}\left(k_{o} c\right) J_{n}^{\prime}(k c) \\
d_{n}=H_{n}^{(1)}(k i c) H_{n}^{\left(1 \gamma^{\prime}\right.}\left(k_{o} c\right)-\sqrt{\frac{\epsilon_{r}}{\mu_{r}}} H_{n}^{(1)}\left(k_{o} c\right) H_{n}^{(1)^{\prime}}(k c)
\end{gathered}
$$

The dual series equations are then converted to a certain canonical form and regularized by the Riemann Hilbert Problem technique. The static part of the scattering operator is inverted analytically and the remaining part is further inverted numerically by all accurate algorithm. This partial inversion of the scattering operator finally gives a matrix equation. Resulting matrix equation is Fredholm 2nd kind, so the convergence and accuracy is 
guarenteed and it is possible to obtain a solution with any desired accuracy [2].

\section{Numerical Results}

The effect of the radome on the radiation pattern of a circular reflector antenna is verified by the present method. The far field radiation patteru is obtained for the thin-lossless dielectric radome material. It can be secn from Figure 2 that the transmission coefficient from a dielectric slab remains almost unity for the halfwavelength of the radome thickness for small incident angles. Figure 3 shows that the radiation pattern changes in form due to radome reflections but for the selection of radome thickness as a half of the wavelength in the dielectric medium, then the distortion in the radiation pattern is minimized. The free space radiation pattern of a circular reflector antenna was also compared by the high frequency solution of Jull and Suedan in [3].

\section{Conclusions}

Complex Source-Dual Series approach is applied to a radome covered circular reflector antenna which is excited by a feed has a directive radiation pattern. The present accurate results can be thought as a reliable data for the validity of aproximate solutions. Further, lossy case and the multi layer rarlome problems can be solved by the same method.

\section{Acknowledgement}

This research was sponsored by NATO's Scientific Affairs Division in the framework of the science for stability programme.

\section{References}

[1] L.B. Felsen " Complex source point solutions of the field equations and their relation to the propagation and scattering of Ganssian beams," Symp. Math., vol.18, pp 39-56, 1975

[2] A.I. Nosich "Green's Function-Dual Series Approach in wave scattering by combined resonant scatterers," in M.Hashimoto, M.Idenen and O.A. Tretyakov (eds.), Analytical and Numerical Methods in EM Wave Theory, Tokyo, Science Honse, 1992.

[3] T. Oğuzer, A. Altıntaş and A.I. Nosich "Analysis of circular reflectors by complex source-dual series approach", in IEEE AP-S International Symp., proc, vol.2, pp. 922-925, 1993 . 

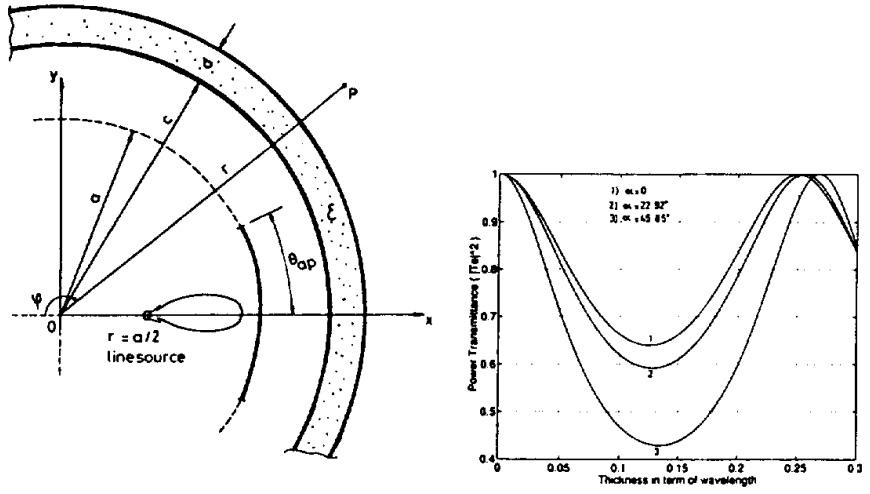

Figure 1: a) Circular reflector and radome geometry b) Power transmission coefficient of a dielectric $\operatorname{slab}\left(\epsilon_{r}=4\right)$

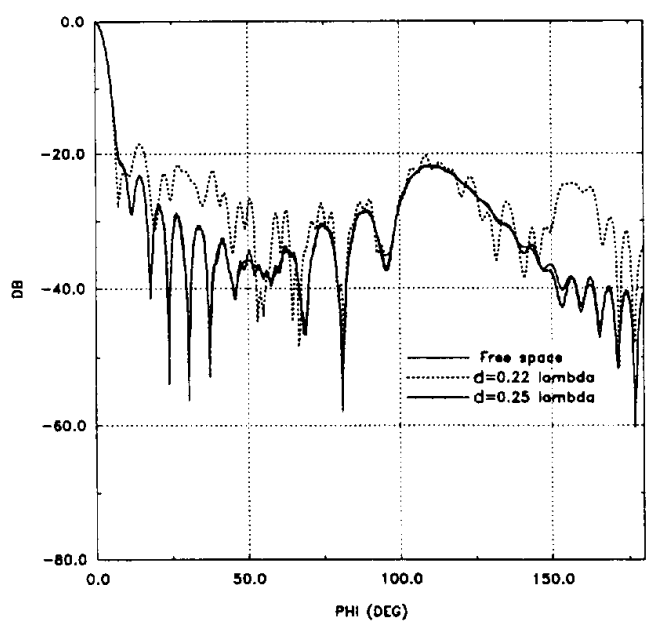

Figure 2: Radiation pattern of a circular reflector antenna in the presence of a co-axial cylindrical radome $(\mathrm{ka}=62.8, \mathrm{~kb}=2.6$, $\left.\mathrm{kc}=100, \epsilon_{\mathrm{r}}=\mathbf{4}\right)$ 